\title{
Hierarchical Honeycomb Lattice Metamaterials with Improved Thermal Resistance and Mechanical Properties
}

\author{
Yanyu Chen ${ }^{\dagger}$, Zian Jia ${ }^{\dagger}$, and Lifeng Wang ${ }^{\dagger *}$ \\ ${ }^{\dagger}$ Department of Mechanical Engineering, State University of New York at Stony Brook, \\ Stony Brook, New York 11794, USA
}

\begin{abstract}
Recent advances in the defense, aerospace, and energy industries have triggered tremendous demand for multifunctional materials featuring lightweight, load-carrying capacity, and thermal resistance. Metamaterials with artificially engineered architectures can be exploited to provide a compelling combination of these properties. Here we report a group of hierarchically architected metamaterials constructed by replacing cell walls of regular honeycombs with hexagonal, kagome, and triangular lattices, respectively. Our numerical and analytical studies indicate that the introduction of structural hierarchy in regular honeycombs results in improved heat resistance and thermal anisotropy. These thermal properties can be controlled by tailoring two geometric parameters of the hierarchical honeycombs. We also predict that the hierarchical honeycombs with kagome and triangular lattices exhibit enhanced mechanical properties. Our analysis reveals that the integrated thermal mitigation and loadcarrying capacity of the hierarchical honeycombs are attributed to the introduction of structural hierarchy. The hierarchical metamaterials reported here provides new opportunities to design multifunctional materials that are promising for various engineering applications.
\end{abstract}

KEYWORDS: metamaterials, hierarchical, honeycombs, heat conduction, thermal anisotropy, multifunctional.

\section{* Corresponding author}

E-mail: lifeng.wang@ stonybrook.edu 


\section{INTRODUCTION}

Metamaterials are artificially engineered materials whose physical properties are dictated by the inherent architectures rather than their compositions. These novel materials have attracted intensive research interests because they enable a combination of unusual properties that cannot be achieved in natural materials. Typical unusual properties range from negative refractive indexes in electromagnetic metamaterials to negative mass density $[1,2]$ and ultrahigh stiffness in mechanical metamaterials.[3-6] For example, recent advances in fabrication techniques have allowed for the fabrication of mechanical metamaterials with rationally controlled topology and cell size.[3-11] These micro-/nanostructured metamaterials demonstrate lightweight and ultrahigh stiffness over a wide range of density. Additionally, other novel physical properties including tunable photonic properties[6] and heat mitigation capability have also been reported in the architected metamaterials. $[12,13]$ These advances indicate that metamaterials with artificially engineered architectures can be considered as prime candidates for next generation multifunctional materials.

Despite considerable advances and efforts, designing materials that possess integrated properties in various physical domains is still challenging. For example, the structural systems in aerospace engineering require that the structural components should have a combination of lower weight/compactness, enhanced load-carrying capacity, and improved thermal resistance. This requirement poses a great challenge for both engineers and researchers due to the strong coupling among these physical properties in conventional bulk materials. Specifically, high stiffness conflicts with the requirements of lightweight and improved thermal resistance. To this end, lattice materials with different coordinate numbers, such as hexagonal lattice, kagome lattice, and triangular lattice, have been widely deployed due to their lightweight, novel 
thermomechanical properties, and energy absorption capability.[14-19] Recently, inspired by the observation that many biological materials have developed multilevel of structural hierarchy enabling the combination of unusual mechanical properties to protect against environmental threats, researchers have introduced structural hierarchy into the conventional lattice materials.[20-25] For example, by replacing the cell walls of regular honeycombs with kagome and triangular lattices, it is theoretically demonstrated that the stiffness of the hierarchical honeycombs is increased by about two orders of magnitude as compared to that of regular honeycombs.[23] However, the underlying mechanisms responsible for this unusual mechanical property are still unrevealed. Although it has been shown that structural hierarchy has significant influences on the transportation process of fractal networks,[26-28] the effect of structural hierarchy on heat conduction of hierarchical honeycombs is not fully understood.

Here, we propose a hierarchical metamaterial concept aiming at addressing the conflicts between the material properties in mechanical domain and thermal domain. The proposed hierarchical architectures are constructed by replacing the cell walls of the regular honeycombs with hexagonal, kagome, and triangular lattices, respectively (referred to as hexagonal, kagome, and triangular hierarchical honeycombs for simplicity in the following). We investigate the heat conduction and mechanical response of the hierarchical honeycombs using the finite element method. We will show that the introduction of structural hierarchy in regular honeycombs gives rise to improved thermal resistance and thermal anisotropy. More important, the proposed hierarchical honeycombs also exhibit enhanced mechanical properties, thereby providing opportunities to design architected materials with simultaneous improvement in heat mitigation and load-carrying capacity. 


\section{MODELS AND METHODS}

\subsection{Characterization of the hierarchical honeycombs}

(a)

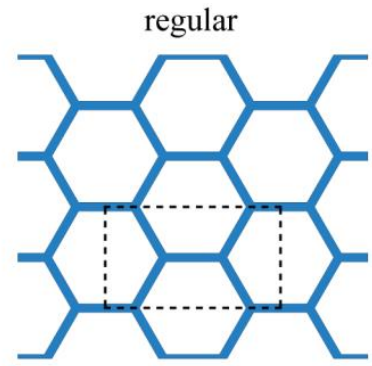

(b)

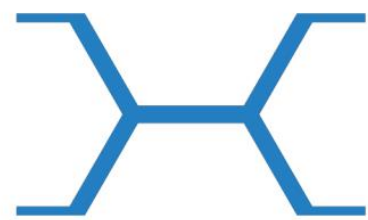

(c)

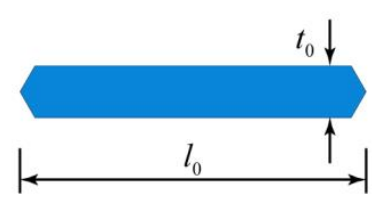

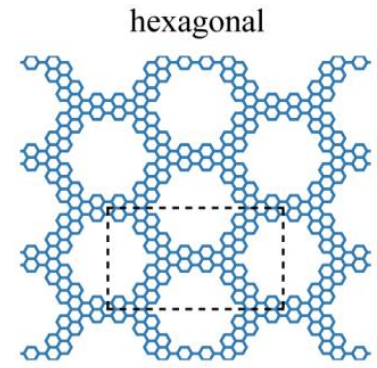
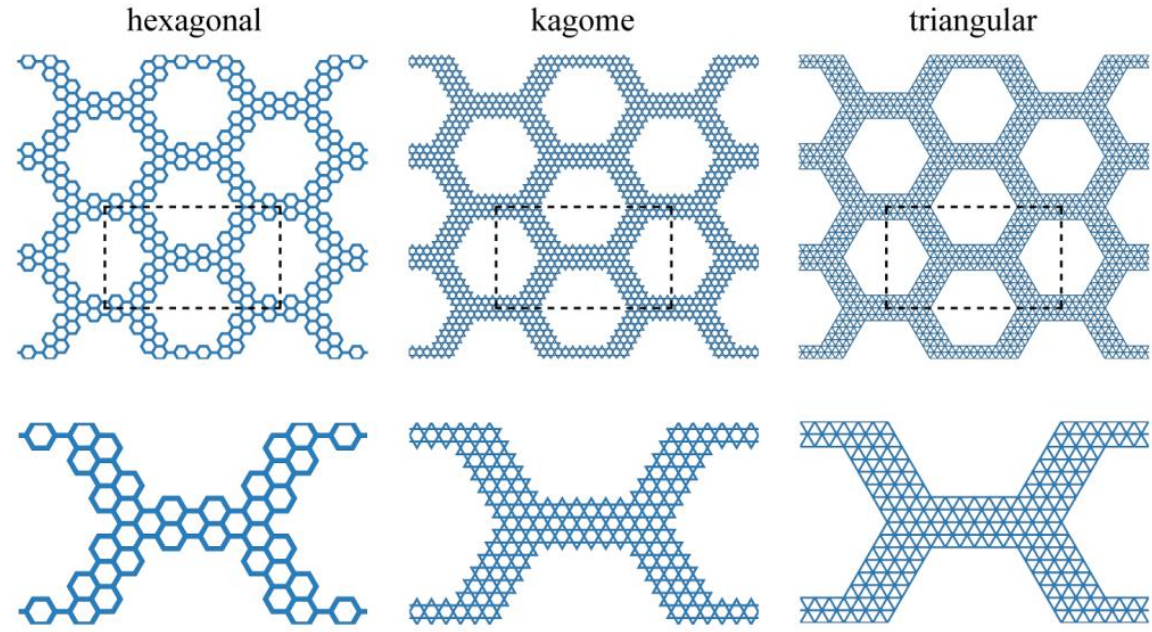

-

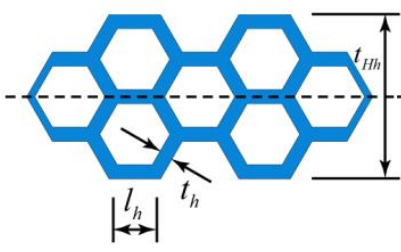

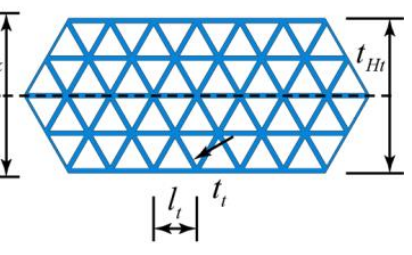

Figure 1. Schematic illustrations of regular honeycomb and hierarchical honeycombs. (a) Regular honeycomb and hierarchical honeycombs consisting of $2 \times 3$ representative volume elements. The dash lines indicate the representative volume element of each structure. (b) Representative volume elements of each structure and (c) the corresponding cell walls. Here $\gamma$ $=1 / 8, N=1$, and relative density of each structure is 0.16 .

Hexagonal hierarchical honeycombs are constructed by replacing the cell walls of regular honeycombs with hexagonal lattice (Figure 1). For the purpose of fair comparisons, kagome and triangular hierarchical honeycombs are subsequently obtained by connecting the midpoints and vertices of the hexagonal lattice, respectively. The proposed hexagonal hierarchical honeycombs 
are characterized by two geometric parameters, hierarchical length ratio, $\gamma=l_{h} / l_{0}$, and the number of hexagonal lattice away from the central axis, $N$, where $l_{0}$ and $l_{h}$ are the length of cell walls of regular honeycomb and hexagonal lattice, respectively. The length and thickness of the hexagonal, kagome, and triangular lattices are determined by mass equivalence between regular honeycombs and hierarchical honeycombs (Figure 1 (c)). For the case of hexagonal hierarchical honeycombs, the mass equivalence gives

$t_{0} l_{0}-\frac{t_{0}^{2}}{2 \sqrt{3}}=3 P\left(t_{h} l_{h}-\frac{t_{h}^{2}}{2 \sqrt{3}}\right)$

where $t_{0}$ and $t_{h}$ are the cell wall thickness of regular honeycomb and hexagonal lattice, respectively; $P$ is the number of hexagonal lattice with one half thickness and is determined by $\gamma$ and $N$.

As a result, the thickness and length of the hexagonal lattice can be calculated as

$$
\frac{t_{h}}{l_{h}}=\sqrt{3}\left(1-\sqrt{1-\frac{2}{3 \sqrt{3} P \gamma^{2}}\left[\frac{t_{0}}{l_{0}}-\frac{1}{2 \sqrt{3}}\left(\frac{t_{0}}{l_{0}}\right)^{2}\right]}\right)
$$

Similarly, the thickness and length of kagome lattice and triangular lattice are given by

$$
\begin{aligned}
& \frac{t_{k}}{l_{k}}=\frac{\sqrt{3}}{2}\left(1-\sqrt{1-\frac{16}{3 \sqrt{3} Q \gamma^{2}}\left[\frac{t_{0}}{l_{0}}-\frac{1}{2 \sqrt{3}}\left(\frac{t_{0}}{l_{0}}\right)^{2}\right]}\right) \\
& \frac{t_{t}}{l_{t}}=\frac{1}{\sqrt{3}}\left(1-\sqrt{1-\frac{4 \sqrt{3}}{3 R \gamma^{2}}\left[\frac{t_{0}}{l_{0}}-\frac{1}{2 \sqrt{3}}\left(\frac{t_{0}}{l_{0}}\right)^{2}\right]}\right)
\end{aligned}
$$


where $t_{k}$ and $l_{k}$ are the thickness and length of cell walls of kagome lattice; $t_{t}$ and $l_{t}$ are the thickness and length of cell walls of triangular lattice, respectively; $Q$ and $R$ are the number of kagome and triangular lattices, respectively.

Here, the side length of regular honeycombs $l_{0}$ is set to $1 \mathrm{~cm}$. Then for a given relative density, $\gamma$, and $N$, the side length and thickness of each lattice can be determined from Eq.(2)Eq.(4), respectively. The proposed regular and hierarchical honeycombs consist of a type of Aluminum, whose thermal properties are characterized by heat capacity $900[\mathrm{~J} /(\mathrm{kg} \cdot \mathrm{K})]$ and thermal conductivity $238[\mathrm{~W} /(\mathrm{m} \cdot \mathrm{K})]$, and mechanical properties are characterized by Young's modulus $70 \mathrm{GPa}$, Poisson's ratio 0.33 and yield strength $148 \mathrm{MPa}$.[29] In addition, the density of solid Aluminum is $2700 \mathrm{~kg} / \mathrm{m}^{3}$.

\subsection{Calculation of effective thermal conductivity}

\subsubsection{Numerical modeling}

In view of the fact that the thermal conductivity of aluminum is about four orders of magnitude larger than that of air, we assume that heat conduction only occurs in the solid cell walls of the hierarchical honeycombs. Here we only focus on the in-plane heat conduction in the proposed hierarchical honeycombs, although the out-of-plane property can be investigated by following the same procedure. To calculate the in-plane effective thermal conductivity (ETC) of the hierarchical honeycombs, we impose a temperature gradient across the representative volume element (RVE) along $x$ and $y$ directions and then compute the heat flux distribution. Specifically, heat periodic boundary conditions are applied along both $x$ and $y$ directions such that

$$
-\mathbf{n}_{i} \cdot \mathbf{q}_{i}=\mathbf{n}_{j} \cdot \mathbf{q}_{j}
$$


where $\mathbf{n}$ is the normal vector of the boundary, $\mathbf{q}$ is the heat flux vector, $i$ and $j$ indicate the source boundary and destination boundary, respectively. The heat flux distribution of the hierarchical honeycombs is computed by solving the governing equation of heat transfer in solids,

$\rho C_{p} \frac{\partial T}{\partial t}+\nabla \cdot(-k \nabla T)=Q$

where $\rho$ is the solid density, $C_{p}$ is the solid heat capacity at constant pressure, $k$ is the solid thermal conductivity, and $Q$ is the heat source. The first term on the left-hand-side is zero for a static heat conduction problem. Then the ETC of the hierarchical honeycombs along each direction is evaluated using Fourier's law

$$
\frac{\iint_{S} q}{A_{e f f}}=k_{e f f} \frac{T_{h}-T_{c}}{L_{e f f}}
$$

where $A_{\text {eff, }}, L_{\text {eff, }}$, and $k_{\text {eff }}$ are the effective area, length, and thermal conductivity of the hierarchical honeycombs, respectively; $S$ is the solid area in the middle plane; $T_{h}-T_{c}$ is the applied temperature difference along each direction.

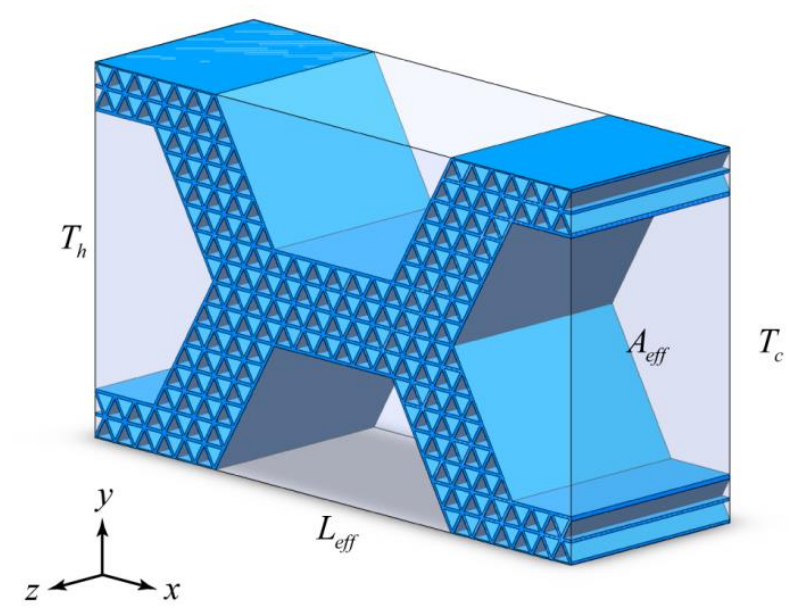


Figure 2. Schematic illustration of the numerical modeling of effective thermal conductivity along $x$ direction. Here the geometry is a triangular hierarchical honeycomb, where $A_{f f}$ and $L_{\text {eff }}$ are the effective cross-sectional area and effective length of the bounding box, respectively; $T_{h^{-}}$ $T_{c}$ is the applied temperature difference along $x$ direction.

\subsubsection{Approximated analytical solutions}

To validate the proposed numerical modeling framework, we also develop an approximate analytical solution to predict the in-plane ETC of hierarchical honeycombs. It has been shown that the ETC of regular hexagonal honeycombs approaches the Hashin-Shtrikman (H-S) upper bound,[30-32] as

$$
\frac{k_{r}}{k_{s}}=\frac{\bar{\rho}_{r}}{2-\bar{\rho}_{r}}
$$

where $k_{r}$ and $k_{s}$ are the thermal conductivity of regular honeycombs and solid constituent

material, respectively; $\bar{\rho}_{r}=\frac{1}{3}\left(2 \sqrt{3}-\frac{t_{0}}{l_{0}}\right) \frac{t_{0}}{l_{0}}$ is the relative density of regular honeycombs. The ETC of hierarchical honeycombs can be determined by first calculating the ETC of the lattice and then substituting it into the H-S upper bound of the equivalent hierarchical honeycombs. The ETC for hexagonal hierarchical honeycombs is calculated as

$$
\frac{k_{H h}}{k_{s}}=\frac{\bar{\rho}_{H h} \cdot \bar{\rho}_{h}}{\left(2-\bar{\rho}_{H h}\right)\left(2-\bar{\rho}_{h}\right)},
$$

where $\bar{\rho}_{H h}=\frac{1}{3}\left(2 \sqrt{3}-\frac{t_{H h}}{l_{H h}}\right) \frac{t_{H h}}{l_{H h}}$ and $\bar{\rho}_{h}=\frac{1}{3}\left(2 \sqrt{3}-\frac{t_{h}}{l_{h}}\right) \frac{t_{h}}{l_{h}}$ are the relative densities of equivalent hexagonal hierarchical honeycombs and hexagonal lattice; $k_{H h}$ and $k_{h}$ are ETC of the equivalent 
hexagonal hierarchical honeycombs and hexagonal lattice, respectively. Here $l_{H h}=l_{0}$, $t_{H h}=\sqrt{3} N l_{h}+t_{h}$. Similarly, the ETC for kagome and triangular hierarchical honeycombs are given by

$\frac{k_{H k}}{k_{s}}=\frac{\bar{\rho}_{H k} \cdot \bar{\rho}_{k}}{\left(2-\bar{\rho}_{H k}\right)\left(2-\bar{\rho}_{k}\right)}$,

$\frac{k_{H t}}{k_{s}}=\frac{\bar{\rho}_{H t} \cdot \bar{\rho}_{t}}{\left(2-\bar{\rho}_{H t}\right)\left(2-\bar{\rho}_{t}\right)}$,

where $\bar{\rho}_{H k}$ and $\bar{\rho}_{H t}$ the relative densities of equivalent kagome and triangular hierarchical honeycombs, respectively; $k_{H k}$ and $k_{H t}$ are ETC of the equivalent kagome and triangular hierarchical honeycombs, respectively. Here $\bar{\rho}_{H k}=\bar{\rho}_{H t}=\bar{\rho}_{H h}, \bar{\rho}_{k}=\sqrt{3}\left(1-\frac{1}{\sqrt{3}} \frac{t_{k}}{l_{k}}\right) \frac{t_{k}}{l_{k}}$, and $\bar{\rho}_{t}=2 \sqrt{3}\left(1-\frac{\sqrt{3}}{2} \frac{t_{t}}{l_{t}}\right) \frac{t_{t}}{l_{t}}$

For the purpose of comparison, here we also present the analytical solutions of ETC of regular honeycombs along both $x$ and $y$ directions,[33] which are given by

$\frac{k_{r x}}{k_{s}}=\frac{k_{r y}}{k_{s}}=\frac{\bar{\rho}_{r}}{2}$,

where $k_{r x}$ and $k_{r y}$ are the ETC of regular honeycombs along $x$ and $y$ directions, respectively.

\subsection{Modeling the mechanical response of hierarchical honeycombs}

To study the mechanical response of periodic structures, as long as no microscopic bifurcation happens[34], modeling on a smallest repeating RVE,[35] together with periodic boundary 
conditions is computationally efficient. For aluminum honeycomb, it has been shown that its post-yield behavior is localized in an asymmetric shear band.[36] However, we focus on strain region $\varepsilon=0 \sim 0.1$ in this work, and suppose the load is quasi-static and material is nearly-perfect, in which case choosing the "primitive cell" as the RVE (Figure 3) is reasonable.

When the RVE is subjected to a macroscopic deformation gradient, $\mathbf{F}$, periodic boundary conditions are applied on the sides of RVE such that [37-39]

$\mathbf{u}(B)-\mathbf{u}(A)=(\mathbf{F}-\mathbf{I})\{\mathbf{X}(B)-\mathbf{X}(A)\}=\mathbf{H}\{\mathbf{X}(B)-\mathbf{X}(A)\}$

where $A$ and $B$ are two points periodically located on the two sides of the RVE; $\mathbf{u}$ denotes displacement, $\mathbf{X}$ denotes the position in reference configuration, $\mathbf{F}$ is the deformation gradient tensor, and $\mathbf{H}=\mathbf{F}-\mathbf{I}$ is the macroscopic displacement gradient tensor. Note that for the plane strain problem in this work, the displacement gradient is reduced to a $2 \times 2$ matrix

$\mathbf{H}=\left[\begin{array}{ll}H_{11} & H_{12} \\ H_{21} & H_{22}\end{array}\right]=\left[\begin{array}{cc}F_{11}-1 & F_{12} \\ F_{21} & F_{22}-1\end{array}\right]$

In finite element implementation, the components of $\mathbf{H}$ are assigned to be the displacement components of two reference nodes in the RVE. After that, we use the principle of virtual work[37-39] to capture the macroscopic mechanical response of the RVE under various loading conditions.

(a)

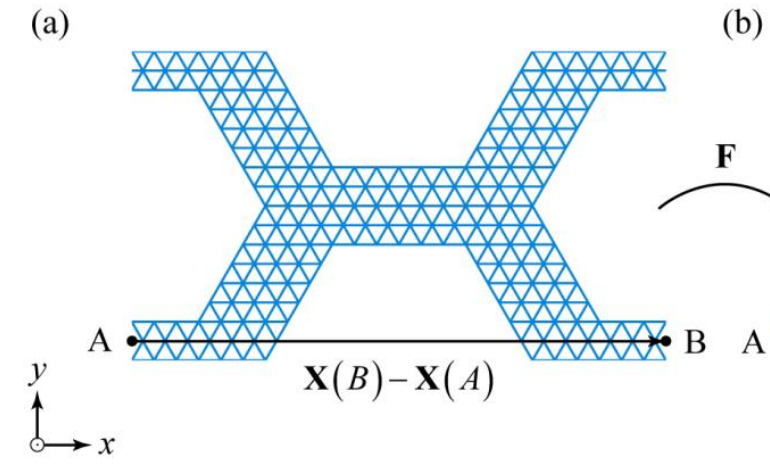

(b)

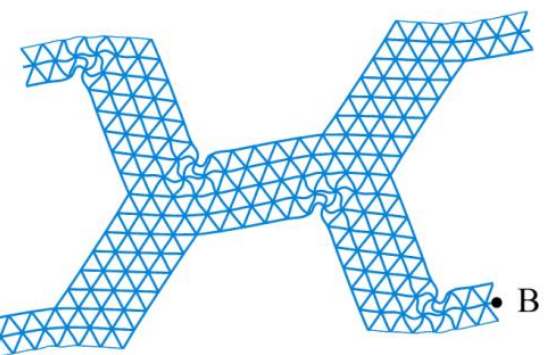


Figure 3. Schematic illustration of periodic boundary conditions on the representative volume element of the triangular hierarchical honeycomb. (a) Undeformed and (b) deformed states. Here $A$ and $B$ are two points periodically located on the two sides of the RVE.

\section{RESULTS AND DISCUSSION}

To validate the proposed numerical modeling approach of heat conduction, we compare the simulated ETC of regular honeycombs and hierarchical honeycombs with those obtained from analytical solutions. Here we set $\gamma=1 / 8, N=1$, and the relative density under investigation is varied from 0.023 to 0.316 . For the regular honeycombs (Figure 4), the simulated ETC along both $x$ and $y$ directions agree perfectly with those predicted by H-S upper bound, except that there is discrepancy between the simulated ETC and the approximate analytical solutions, Eq.(12), for relative density larger than 0.1. This indicates that it is reasonable to use H-S upper bound to evaluate the ETC of each level of the hierarchical honeycombs. Excellent agreements between the simulated ETC and the analytical solutions are also observed for hierarchical honeycombs, except that there is some discrepancy between the simulated ETC along $x$ and $y$ directions. For example, the ETC along $y$ direction for the kagome hierarchical honeycombs with a relative density of 0.022 is about $12 \%$ lower than that along $x$ direction. This discrepancy implies that thermal anisotropy exists in hierarchical honeycombs, which cannot be predicted by the approximate analytical solutions. We therefore believe that the proposed numerical modeling framework is an effective approach that can be exploited to investigate heat conduction and explore unusual thermal properties in hierarchical honeycombs. 

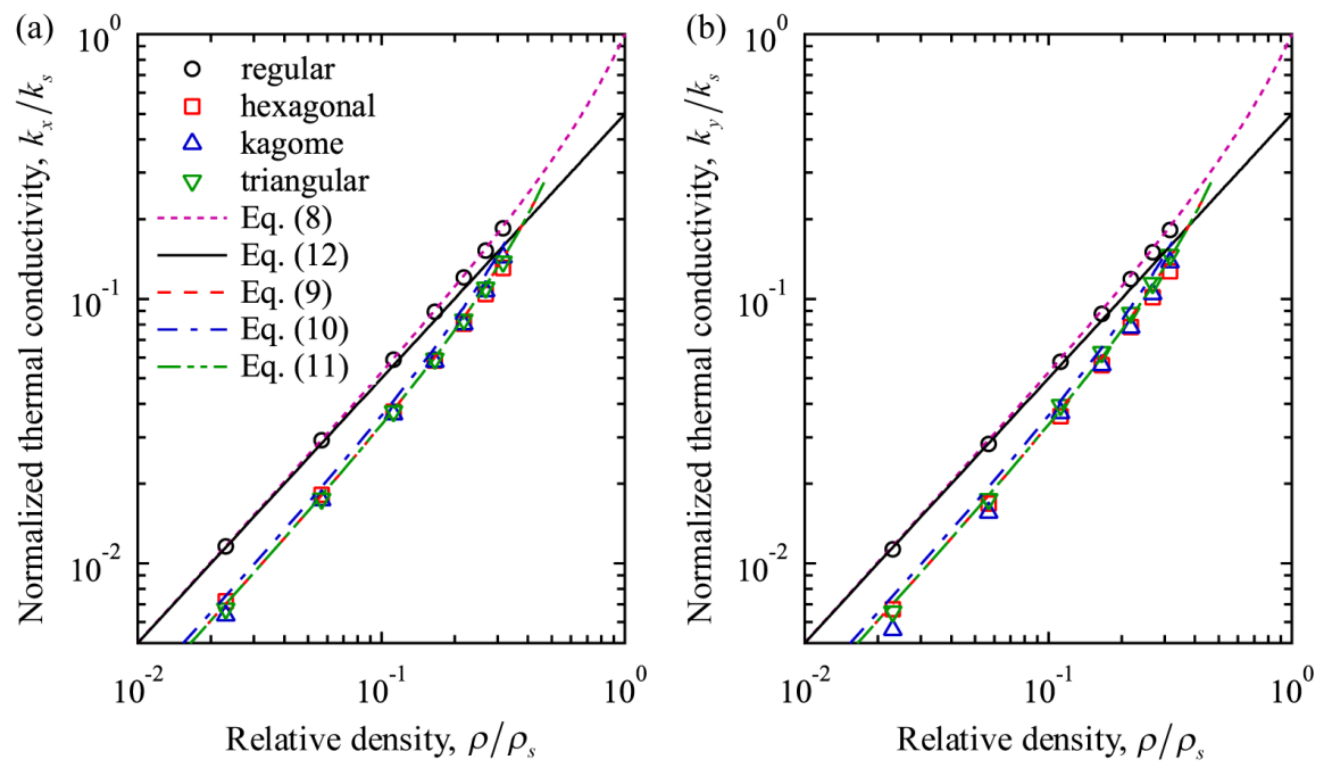

Figure 4. In-plane effective thermal conductivity of hierarchical honeycombs as a function of relative density. (a) Along $x$ direction and (b) along $y$ direction. Here $\gamma=1 / 8$ and $N=1$.

Effects of Geometric Features on ETC. In addition to the thermal anisotropy characteristic, we note that hierarchical honeycombs exhibit lower ETC compared with those of regular honeycombs (Figure 4). These unusual thermal properties are attributed to the introduction of structural hierarchy in the regular honeycombs, thereby changing the thermal resistance. To further understand this, we examine the effects of two geometric features of the hierarchical honeycombs, $\gamma$ and $N$, on the evolution of ETC and the associated thermal anisotropy. Here the relative density of hierarchical honeycombs is set to 0.16 . We note that when the hierarchical length ratio gradually decreases from $1 / 2$ to $1 / 11$, hierarchical honeycombs exhibit much lower ETC along both $x$ and $y$ directions as compared to that of regular honeycombs (Figure 5). Notably, for a given hierarchical length ratio, the minimum $N$ gives rise to lowest ETC. For example, the ETC, normalized by $k_{s}$, along $x$ direction for hexagonal, kagome, and triangular 
hierarchical honeycombs $(\gamma=1 / 11)$ are $0.058,0.058$, and 0.059 , respectively, which are about $65 \%$ of the ETC of regular honeycombs. We also note that triangular hierarchical honeycombs show the lowest ETC for a given $\gamma$ and $N$, by comparing the ETC along both $x$ and $y$ directions. These results not only suggest that structural hierarchy can improve the thermal resistance, but also indicate that desired ETC can be achieved by tailoring the two critical geometric parameters of the hierarchical honeycombs.
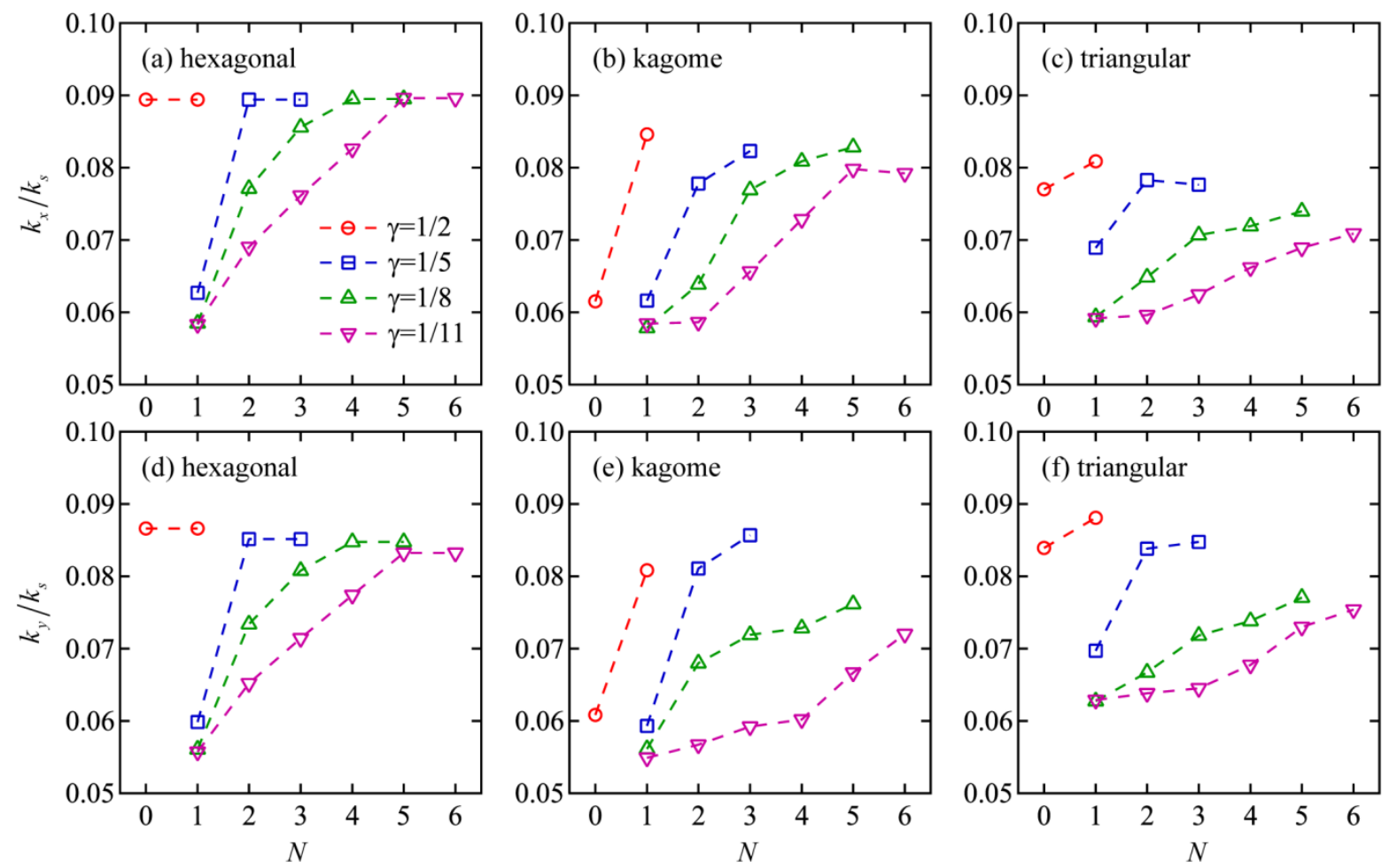

Figure 5. Effects of hierarchical length ratio and number of lattices on the in-plane normalized thermal conductivity. (a)-(c): Normalized thermal conductivity along $x$ direction and (d)-(f) along $y$ direction for hexagonal, kagome, and triangular hierarchical honeycombs, respectively. The relative densities of regular and hierarchical honeycombs are 0.16 . 
It is noticeable from Figure 5 that the discrepancy of ETC along $x$ and $y$ directions persists for the hierarchical honeycombs with different $\gamma$ and $N$. To quantitatively illustrate this, we plot in Figure 6 the thermal anisotropy of the three types of hierarchical honeycombs. Here thermal anisotropy is defined as the ratio of ETC along $x$ direction to that along $y$ direction. For hexagonal hierarchical honeycombs, ETC along $y$ direction is slightly higher than that along $x$ direction. By contrast, triangular hierarchical honeycombs exhibit an opposite trend. Kagome hierarchical honeycombs exhibit fluctuated thermal anisotropy, strongly depending on $\gamma$ and $N$. These findings suggest that regular honeycombs can be considered as isotropic in terms of ETC, whereas heat conduction in hierarchical honeycombs is strongly direction-dependent. Again, this unusual thermal anisotropy is also attributed to the introduction of structural hierarchy to the regular honeycombs. While the different thermal response among these hierarchical honeycombs are intrinsically dictated by the lattice with different geometric features and coordinate numbers.
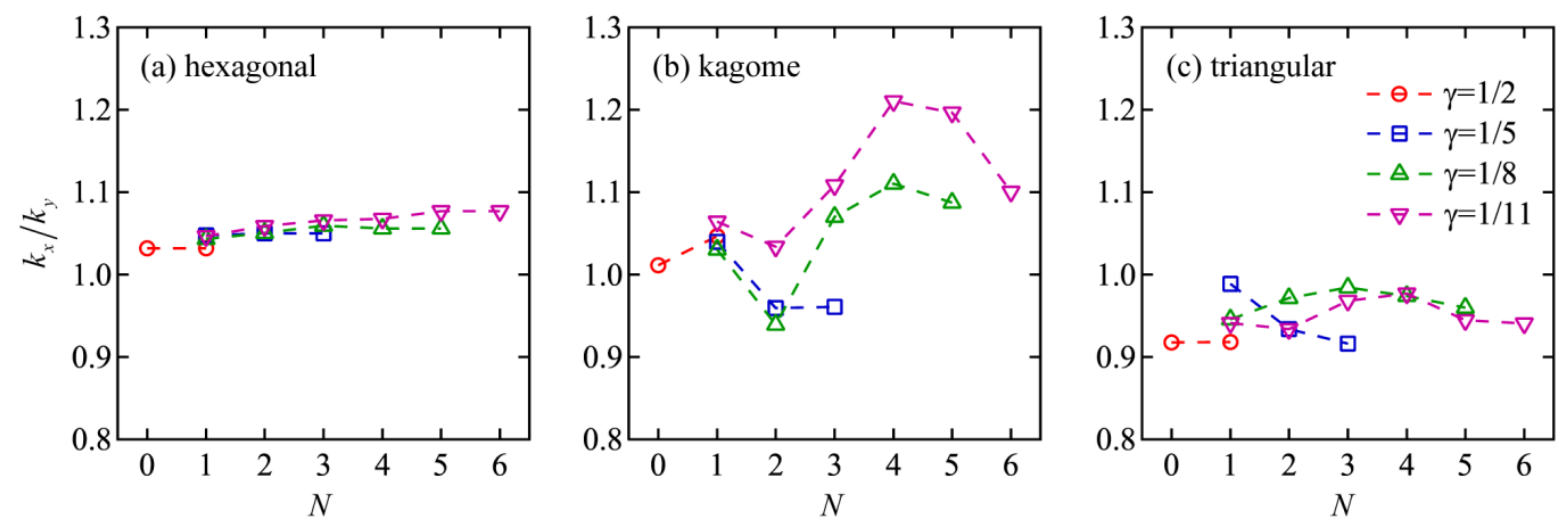

Figure 6. In-plane thermal anisotropy of hierarchical honeycombs. (a)-(c) hexagonal, kagome, and triangular hierarchical honeycombs. The relative densities of regular and hierarchical honeycombs are 0.16 . 
Mechanical Response of Hierarchical Honeycombs. Heat conduction analysis indicates that the proposed hierarchical honeycombs can exhibit thermal anisotropy and improved thermal resistance, which is attributed to the structural hierarchy and intrinsically dictated by the architectures.

To demonstrate our metamaterial design concept, we now examine the mechanical response of the hierarchical honeycombs under uniaxial compression along $x$ and $y$ directions. Here the geometric parameters of the hierarchical honeycombs are given by $\gamma=1 / 8$ and $N=1$. Figure 7 (a) and (b) present the stress-strain relations for regular and hierarchical honeycombs at $\rho / \rho_{s}=0.16$. Along each direction, kagome and triangular hierarchical honeycombs exhibit higher stiffness and strength compared with regular honeycombs. The stiffness along $x$ and $y$ directions are almost the same for each structure, whereas the strength along $x$ direction are higher than those along $y$ direction, suggesting that the hierarchical honeycombs can provide better load resistance along $x$ direction at the same density. Apparently, the stress-strain curves exhibit an initially linearly-elastic and later a nonlinear behavior for the regular honeycombs and hierarchical honeycombs (Figure 7 (a)). Note that the critical aspect ratio for elastic buckling to happen before plastic collapse is given by $(t / l)_{c r i t}=2 \sqrt{3} \sigma_{y s} / E_{s}$, where $\sigma_{y s}$ and $E_{s}$ are the yield strength and Young's modulus of Aluminum.[40] The critical aspect ratio is $6.34 \times 10^{-3}$ for aluminum and is significantly lower than those in our structures. Thusfore, theWe therefore believe that plastic deformation occurs before the elastic buckling in our simulation, which is generalized into three stages:

I. The deformation is purely elastic when the nominal strain is less than 0.005. Compared with regular honeycombs, kagome and triangular hierarchical honeycombs exhibit $\sim 1.8$ and $\sim 2.0$ 
times higher stiffness, respectively, whereas the stiffness of hexagonal hierarchical honeycombs is $\sim 40 \%$ lower than that of regular honeycombs.

II. Plastic deformation arises, leading to a decreasing slope in the stress-strain curve. For regular honeycombs and hexagonal hierarchical honeycombs, plastic deformation is localized in the ligament joints (Figure 7(c) A). This behavior holds till $\varepsilon=0.10$ and the stress slowly increases toward a plateau. By contrast, triangular hierarchical honeycombs are quite different, since the initial plastic deformation is stretch dominated (Figure 7 (c) B) and distributes more globally. Such behavior benefits greater slope in the curve and the yield stress is $\sim 3$ times higher than regular honeycombs. Kagome hierarchical honeycombs deform between stretching and bending, which is characterized by medium stiffness and stress status.

III. Plastic crushing/local damage happens for kagome and triangular hierarchical honeycombs. For kagome hierarchical honeycombs, the sudden drop is caused by local plastic necking (Figure 7(c) C), whereas plastic bending is first induced locally in the triangular lattice, and gradually evolves into rotate center (Figure 7(c) D). 

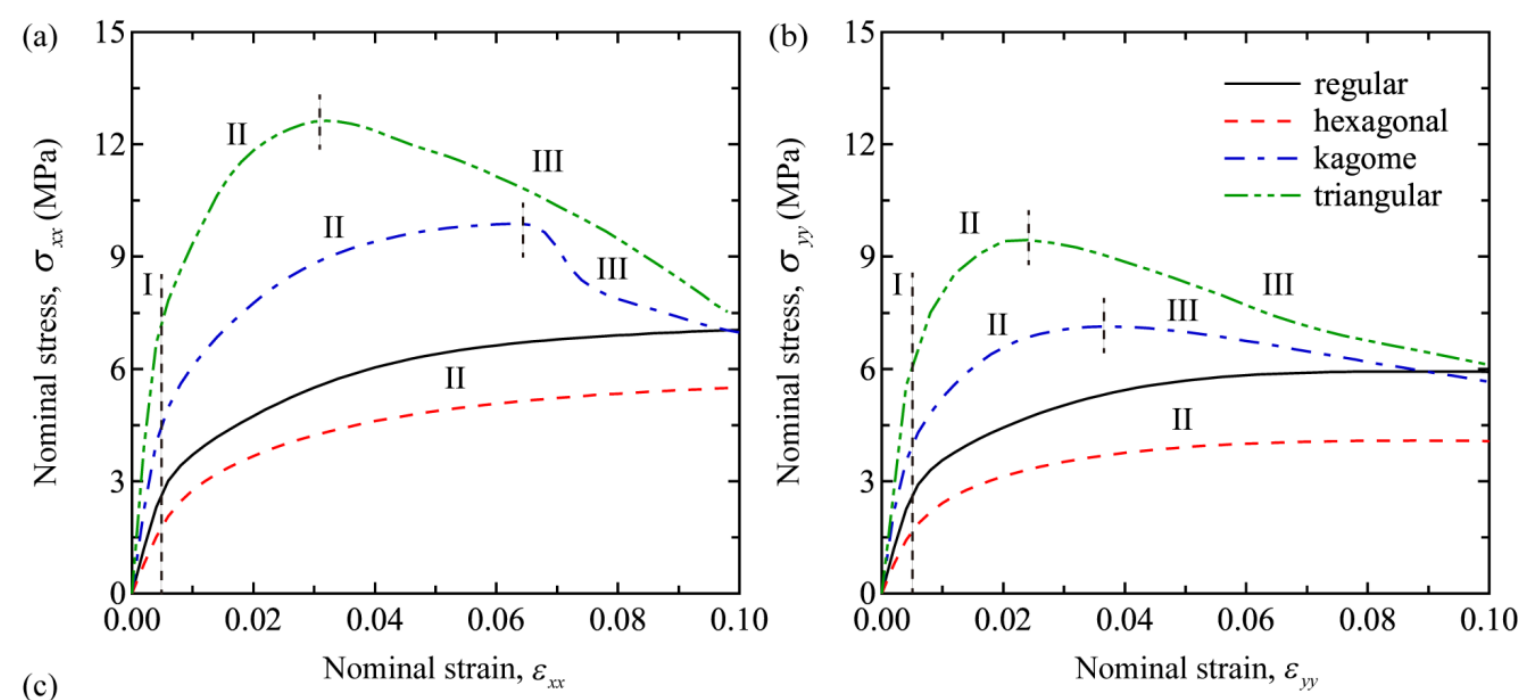

(c)

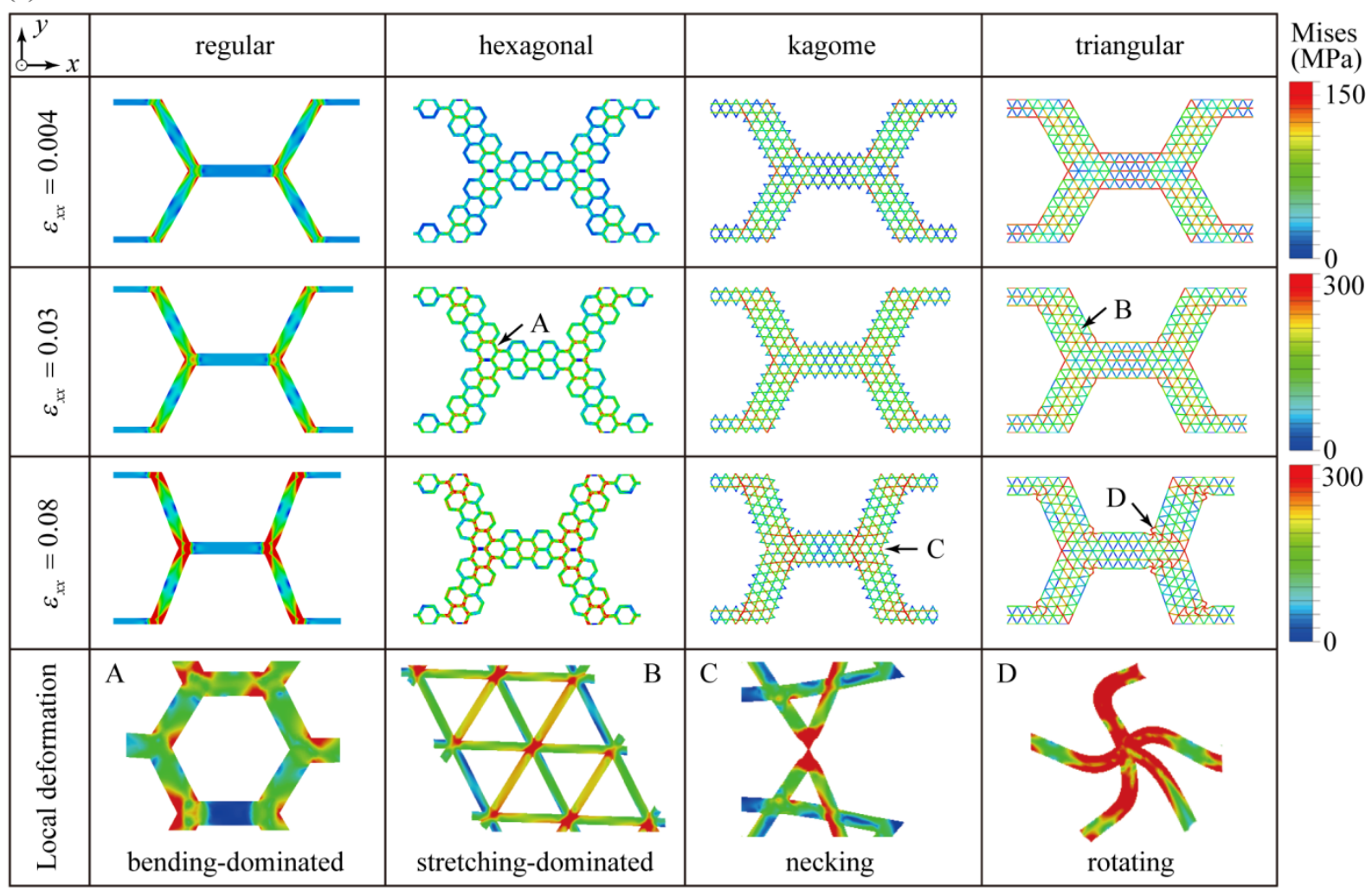

Figure 7. In-plane mechanical response of the hierarchical honeycombs. (a)-(b) Strain-stress curves of the hierarchical honeycombs compressed along $x$ and $y$ directions, respectively; (c) Deformation and distribution of von Mises stress at nominal strain at different strain levels. Here $\gamma=1 / 8, N=1$, and $\rho / \rho_{s}=0.16$. 
To further understand our hierarchical metamaterial concept, we investigate the effect of relative density on the mechanical response of hierarchical honeycombs. Here the relative density is varied from 0.023 to 0.316 , while the two geometric parameters is set to $\gamma=1 / 8$ and $N=1$. For the purpose of comparison, we also plot the stiffness as a function of relative density for regular honeycombs (Figure 8). For regular honeycombs, we notice that the simulated stiffness approach the linear-elastic theory,[40] indicating that our numerical modeling approach can accurately predict the mechanical response of the regular honeycombs. When compressed along a specific direction, hexagonal hierarchical honeycombs have comparable yet slightly lower stiffness than that of regular honeycomb. Remarkably, kagome and triangular hierarchical honeycombs show improved stiffness. For example, at low relative density 0.023 , triangular hierarchical honeycombs exhibit $\sim 160$ times higher stiffness and $\sim 8$ times higher strength than regular honeycombs. To evaluate the mechanical response of hierarchical honeycombs from a macroscopic perspective, we fit the stiffness as a function of relative density using a scaling law, $E_{i} / E_{s}=C\left(\rho / \rho_{s}\right)^{n}$, where $E_{i}$ and $E_{s}$ are the stiffness of hierarchical honeycombs along $x$ or $y$ direction and solid aluminum, respectively; $C$ is geometry-dependent proportionality constant, $n$ is the scaling exponent, and $\rho / \rho_{s}$ is the relative density of each hierarchical honeycomb. For example, the scaling exponents for regular honeycomb, hexagonal, kagome, and triangular hierarchical honeycombs along $x$ directions are 3.01, 2.56, 2.08, and 1.33, respectively, indicating that regular honeycomb, and hexagonal and kagome hierarchical honeycombs exhibit a bending-dominated deformation behavior, whereas triangular hierarchical honeycombs have a stretching-dominated deformation behavior. Similar trend but slightly different fitting exponents are observed in hierarchical honeycombs compressed along $y$ direction, indicating that the 
directionally dependent mechanical response of the hierarchical honeycombs. These results further confirm that introduction of structural hierarchy can significantly improve the stiffness of hierarchical honeycombs.

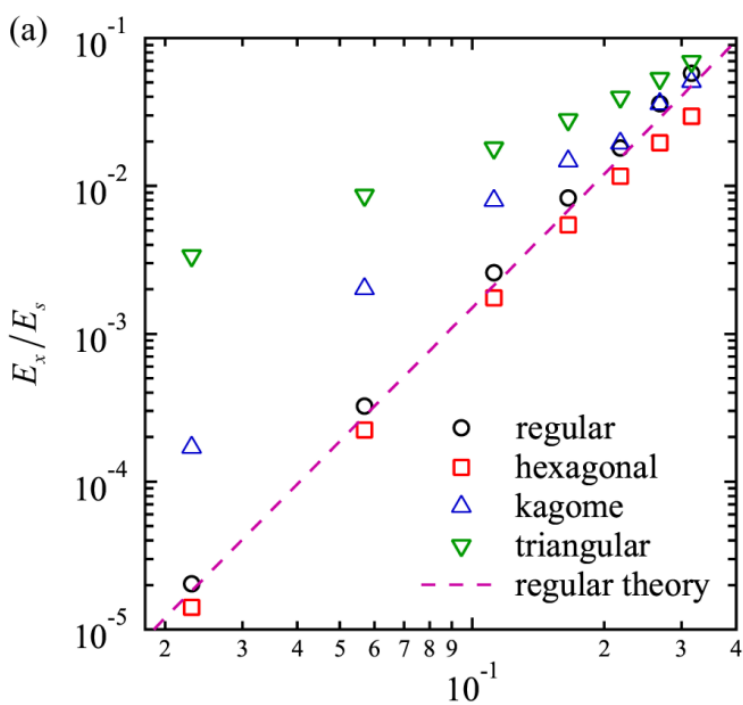

Relative density, $\rho / \rho_{s}$

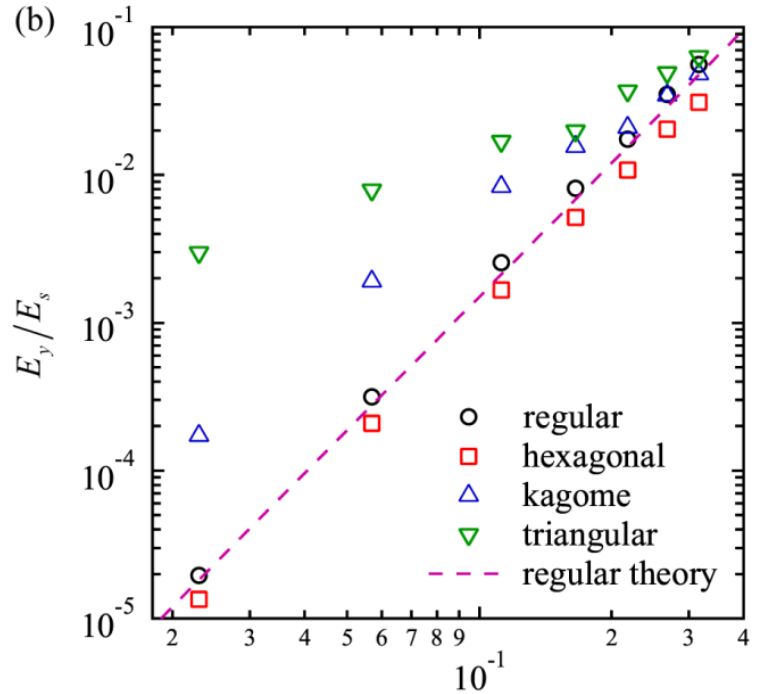

Relative density, $\rho / \rho_{s}$

Figure 8. In-plane stiffness of the hierarchical honeycombs as a function of relative density. (a) Along $x$ direction and (b) along $y$ direction. Here $\gamma=1 / 8$ and $N=1$. The dash line is the analytical solution of regular honeycombs.

Design of Hierarchical Honeycombs with Multifunctionality. Having shown that the proposed hierarchical honeycombs exhibit improved thermal resistance and stiffness, we now proceed to demonstrate that structural hierarchy can be exploited to design multifunctional materials. By combining the simulated ETC and stiffness of the hierarchical honeycombs along $x$ and $y$ directions, we obtain the Ashby-type plots of specific modulus versus ETC (Figure 9). Compared with the regular honeycombs, hexagonal hierarchical honeycombs retain comparable specific modulus but lower ETC along each direction. Remarkably, kagome and triangular 
hierarchical honeycombs can achieve specific stiffness that is two orders of magnitude higher than that of regular honeycombs while having similar ETC. It is also interesting to note that triangular hierarchical honeycombs exhibit a nearly constant specific modulus when the ETC is decreased. These findings imply that hierarchically architected honeycombs hold great potential for engineering applications where thermal mitigation and load-carrying capacity are simultaneously pursued.
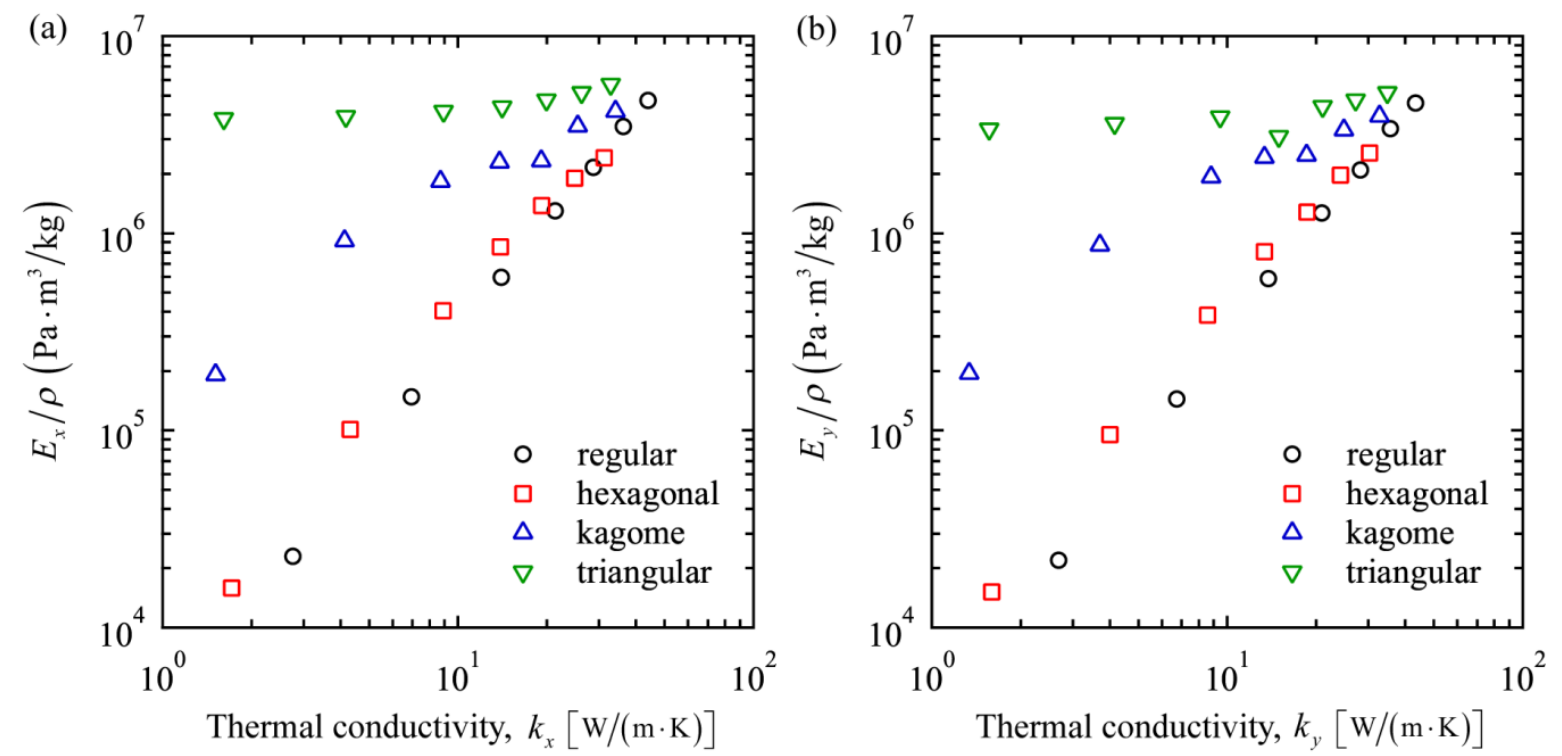

Figure 9. Ashby-type plots for in-plane specific modulus and effective thermal conductivity of regular and hierarchical honeycombs. (a) Along $x$ direction and (b) along $y$ direction. Here $y=1 / 8$ and $N=1$.

\section{CONCLUSION}

In summary, we have numerically demonstrated that structural hierarchy in regular honeycombs can be intentionally exploited to design metamaterials with integrated heat mitigation and loadcarrying capacity. Our results reveal that the improved thermal resistance and thermal anisotropy 
can be controlled by tailoring two geometric features of the hierarchical honeycombs. Moreover, kagome and triangular hierarchical honeycombs also exhibit improved specific stiffness as compared to that of regular honeycombs. We emphasized that these simultaneously achieved thermal and mechanical properties are dictated by the structural hierarchy. From a practical perspective, hierarchical honeycombs have great potential to be employed in the defense, aerospace, energy, and semiconductor industries where lightweight, thermal mitigation, and load-carrying capacity are simultaneously desired. Importantly, the hierarchical metamaterial concept presented here will open new avenues to design architected materials with various combinations of compositions and geometric features, thereby expanding the existing metamaterial design space.

\section{ACKNOWLEGDGEMNETS}

The authors gratefully acknowledge the financial support from the National Science Foundation (CMMI-1462270) and the Office of Naval Research. Y. Chen thanks Mr. Nicholas G. Dou (California Institute of Technology) for stimulating discussion.

\section{REFERENCES}

[1] Ding Y, Liu Z, Qiu C, Shi J. Metamaterial with simultaneously negative bulk modulus and mass density. Phys Rev Lett 2007;99:093904.

[2] Liu X, Hu G, Huang G, Sun C. An elastic metamaterial with simultaneously negative mass density and bulk modulus. Appl Phys Lett 2011;98:251907.

[3] Zheng X, Lee H, Weisgraber TH, Shusteff M, DeOtte J, Duoss EB, et al. Ultralight, ultrastiff mechanical metamaterials. Science 2014;344:1373-7.

[4] Meza LR, Zelhofer AJ, Clarke N, Mateos AJ, Kochmann DM, Greer JR. Resilient 3D hierarchical architected metamaterials. Proc Acad Sci 2015;112:11502-7.

[5] Meza LR, Das S, Greer JR. Strong, lightweight, and recoverable three-dimensional ceramic nanolattices. Science 2014;345:1322-6. 
[6] Chernow V, Alaeian H, Dionne J, Greer J. Polymer lattices as mechanically tunable 3-dimensional photonic crystals operating in the infrared. Appl Phys Lett 2015;107:101905.

[7] Lee JH, Singer JP, Thomas EL. Micro - /Nanostructured Mechanical Metamaterials. Adv Mater 2012;24:4782-810.

[8] Han SC, Lee JW, Kang K. A New Type of Low Density Material: Shellular. Adv Mater 2015;27:5506-11.

[9] Compton BG, Lewis JA. 3D - Printing of Lightweight Cellular Composites. Adv Mater 2014;26:5930-5.

[10] Wang L, Lau J, Thomas EL, Boyce MC. Co - Continuous composite materials for stiffness, strength, and energy dissipation. Adv Mater 2011;23:1524-9.

[11] Lee J-H, Wang L, Boyce MC, Thomas EL. Periodic bicontinuous composites for high specific energy absorption. Nano lett 2012;12:4392-6.

[12] Dou NG, Minnich AJ. Heat conduction in multifunctional nanotrusses studied using Boltzmann transport equation. Appl Phys Lett 2016;108:011902.

[13] Eckel ZC, Zhou C, Martin JH, Jacobsen AJ, Carter WB, Schaedler TA. Additive manufacturing of polymer-derived ceramics. Science 2016;351:58-62.

[14] Evans AG, Hutchinson JW, Fleck NA, Ashby M, Wadley $H$. The topological design of multifunctional cellular metals. Prog Mater Sci 2001;46:309-27.

[15] Wadley HN, Fleck NA, Evans AG. Fabrication and structural performance of periodic cellular metal sandwich structures. Compos Sci Technol 2003;63:2331-43.

[16] Wadley HN. Multifunctional periodic cellular metals. Philos Trans R Soc, A 2006;364:31-68.

[17] Bezazi A, Remillat C, Innocenti P, Scarpa F. In-plane mechanical and thermal conductivity properties of a rectangular-hexagonal honeycomb structure. Compos Struct 2008;84:248-55.

[18] Zheng L, Wu D, Zhou A, Pan B, Wang Y, Wang J. Experimental and Numerical Study on Heat Transfer Characteristics of Metallic Honeycomb Core Structure in Transient Thermal Shock Environments. Int J Thermophys 2014;35:1557-76.

[19] Gu S, Lu T, Evans A. On the design of two-dimensional cellular metals for combined heat dissipation and structural load capacity. Int J Heat Mass Tran 2001;44:2163-75.

[20] Chen Q, Pugno NM. In-plane elastic buckling of hierarchical honeycomb materials. J Mec Theor Appl 2012;34:120-9.

[21] CÃ F, Russell B, Deshpande V, Fleck N. The through-thickness compressive strength of a composite sandwich panel with a hierarchical square honeycomb sandwich core. J Appl Mech 2009;76:061004.

[22] Ajdari A, Jahromi BH, Papadopoulos J, Nayeb-Hashemi H, Vaziri A. Hierarchical honeycombs with tailorable properties. Int J Solids Struct 2012;49:1413-9.

[23] Sun Y, Pugno NM. In plane stiffness of multifunctional hierarchical honeycombs with negative Poisson's ratio sub-structures. Compos Struct 2013;106:681-9.

[24] Zhao L, Zheng Q, Fan H, Jin F. Hierarchical composite honeycombs. Mater Design 2012;40:124-9.

[25] Taylor C, Smith C, Miller W, Evans K. The effects of hierarchy on the in-plane elastic properties of honeycombs. Int J Solids Struct 2011;48:1330-9.

[26] Yu B, Li B. Fractal-like tree networks reducing the thermal conductivity. Phys Rev E 2006;73:066302.

[27] Xu P, Yu B, Yun M, Zou M. Heat conduction in fractal tree-like branched networks. Int J Heat Mass Tran 2006;49:3746-51.

[28] Xu Z, Buehler MJ. Hierarchical nanostructures are crucial to mitigate ultrasmall thermal point loads. Nano lett 2009;9:2065-72.

[29] Gupta N, Iqbal M, Sekhon G. Experimental and numerical studies on the behavior of thin aluminum plates subjected to impact by blunt-and hemispherical-nosed projectiles. Int J Impact Eng 2006;32:192144.

[30] Torquato S, Gibiansky L, Silva M, Gibson L. Effective mechanical and transport properties of cellular solids. Int J Mech Sci 1998;40:71-82. 
[31] Hyun S, Torquato S. Effective elastic and transport properties of regular honeycombs for all densities. J Mater Res 2000;15:1985-93.

[32] Hyun S, Torquato S. Optimal and manufacturable two-dimensional, Kagome-like cellular solids. J Mater Res 2002;17:137-44.

[33] Lu T, Chen C. Thermal transport and fire retardance properties of cellular aluminium alloys. Acta Mater 1999;47:1469-85.

[34] Müller S. Homogenization of nonconvex integral functionals and cellular elastic materials. Archive for Rational Mechanics and Analysis 1987;99:189-212.

[35] Kittel C. Introduction to solid state physics: Wiley; 2005.

[36] Papka SD, Kyriakides S. In-plane compressive response and crushing of honeycomb. J Mech Phys Solids 1994;42:1499-532.

[37] Danielsson M, Parks D, Boyce M. Three-dimensional micromechanical modeling of voided polymeric materials. J Mech Phys Solids 2002;50:351-79.

[38] Danielsson M, Parks DM, Boyce MC. Micromechanics, macromechanics and constitutive modeling of the elasto-viscoplastic deformation of rubber-toughened glassy polymers. J Mech Phys Solids 2007;55:533-61.

[39] Wang L, Boyce MC, Wen CY, Thomas EL. Plastic Dissipation Mechanisms in Periodic Microframe Structured Polymers. Adv Funct Mater 2009;19:1343-50.

[40] Gibson LJ, Ashby MF. Cellular solids: structure and properties. Cambridge university press;; 1997. 